\title{
Helium stars: Towards an understanding of Wolf-Rayet evolution
}

\author{
Liam A. S. McClelland and J. J. Eldridge \\ Department of Physics, University of Auckland \\ email:1mcc054@aucklanduni.ac.nz \\ email:j.eldridge@auckland.ac.nz
}

\begin{abstract}
Recent observational modelling of the atmospheres of hydrogen-free Wolf-Rayet stars have indicated that their stellar surfaces are cooler than those predicted by the latest stellar evolution models. We have created a large grid of pure helium star models to investigate the dependence of the surface temperatures on factors such as the rate of mass loss and the amount of clumping in the outer convection zone. Upon comparing our results with Galactic and LMC WR observations, we find that the outer convection zones should be clumped and that the massloss rates need to be slightly reduced. We discuss the implications of these findings in terms of the detectability of Type Ibc supernovae progenitors, and in terms of refining the Conti scenario.
\end{abstract}

Keywords. stars: evolution, stars: Wolf-Rayet, binaries: general

\section{Introduction}

Wolf-Rayet (WR) stars are massive helium-burning stars that, through strong mass loss, have lost all or most of their hydrogen envelopes leaving a partially or fully exposed helium core. We have generated a grid of pure helium star models at various metallicities and shall only study the evolution from onset of core-helium burning onwards.

\section{Computational method}

Construction of the Models. To investigate the evolution of helium stars, we have constructed a grid of hydrogen-free models. We make use of the Cambridge sTARs evolutionary code. Originally developed by Eggleton (1971), it has been modified by various groups; herein, we employ the version described by Stancliffe \& Eldridge (2009). We make our selection of metallicities based on the expected environments of WR stars: $Z=0.008$ for the Large Magellanic Cloud; $Z=0.014$ and $Z=0.02$ being, respectively, "new" and "old" solar metallicity; and $Z=0.04$, double "old" solar. We have utilised the solar abundance determinations from Grevesse \& Sauval (1998) ("old" solar) and Asplund et al. (2009) ("new" solar). A comparison of evolution between models of "old" and "new" solar compositions shows very little difference, and in light of this, we prefer "old" solar abundances for use in our models.

Mass-loss Scheme. We employ a mass-loss scheme, outlined in Eldridge \& Vink (2006), which is derived from Nugis \& Lamers (2000):

$$
\dot{M}_{Z} \propto \dot{M} \beta\left(\frac{Z}{Z_{\odot}}\right)^{\frac{1}{2}},
$$

where $\dot{M}$ is taken from Eldridge \& Vink (2006), $Z$ is the metallicity of the model, and $Z_{\odot}$ is solar metallicity. To test the effect of varying the mass-loss rate on the evolution of a model, we introduce a parameter, $\beta$. We may use this parameter to estimate the 
evolution of the helium star model if, before the hydrogen envelope were removed, more helium burning had occurred. For example in the case of $\beta=1$, the hydrogen envelope is removed before the beginning of helium burning, so the tracks represent the greatest possible effect of mass loss on the models. Thus, more helium mass would be lost from the WR stars. However for $\beta=0$, the evolution towards the end of the track represents how the star would appear if the hydrogen envelope were removed near the end of helium burning.

Envelope inflation. Massive stars develop an extended envelope structure due to an increase in opacity caused by the iron-opacity peak. At stellar temperatures near the iron-opacity peak $\left(\log T_{\text {eff }} \sim 5.2\right)$, a small convective layer forms above a near-void region of the star. The overall effect is a radiation-driven expansion of this high opacity outer layer; a reduction of the apparent stellar temperature. The effect is more severe with increasing metallicity due to the increased abundance of iron-group elements.

We allow the material in inflated envelope to be inhomogeneous and define a clumping factor, $D$, as described by Gräfener, Owocki \& Vink (2012). To investigate the effect of density inhomogeneities in the inflated envelopes of helium stars, we select two values for our clumping factor: 1 and 10 referring to "unclumped" and "clumped", respectively.

When referring to a particular set of models, we shall label them by the metallicity, $\beta$, and clumping factor like so: $(Z, \beta, D)$. For example, $(0.02,1,1)$ refers to the grid of models with $Z=0.02, \beta=1$, and a normal envelope without the clumping factor to enhance the opacity.

\section{Helium giants \& Wolf-Rayet stars}

We may divide our helium star models into two categories: low mass ("helium-giant type"), and high mass ("Wolf-Rayet type").

Low-mass helium stars. Low-mass $\left(<8 \mathrm{M}_{\odot}\right)$ helium star models evolve as "helium giants". A helium giant has a stellar structure that is analogous to that of a red-giant star: a dense core region with an expansive envelope (see left panel of Fig. 1).

High-mass helium stars. High-mass $\left(>8 \mathrm{M}_{\odot}\right)$ helium star models evolve as "traditional" Wolf-Rayet stars, having characteristic high temperatures due to strong mass loss (Crowther 2007). The structure of a high-mass helium star differs from that of a lowmass helium star by the properties of its envelope: an extended region of near-constant
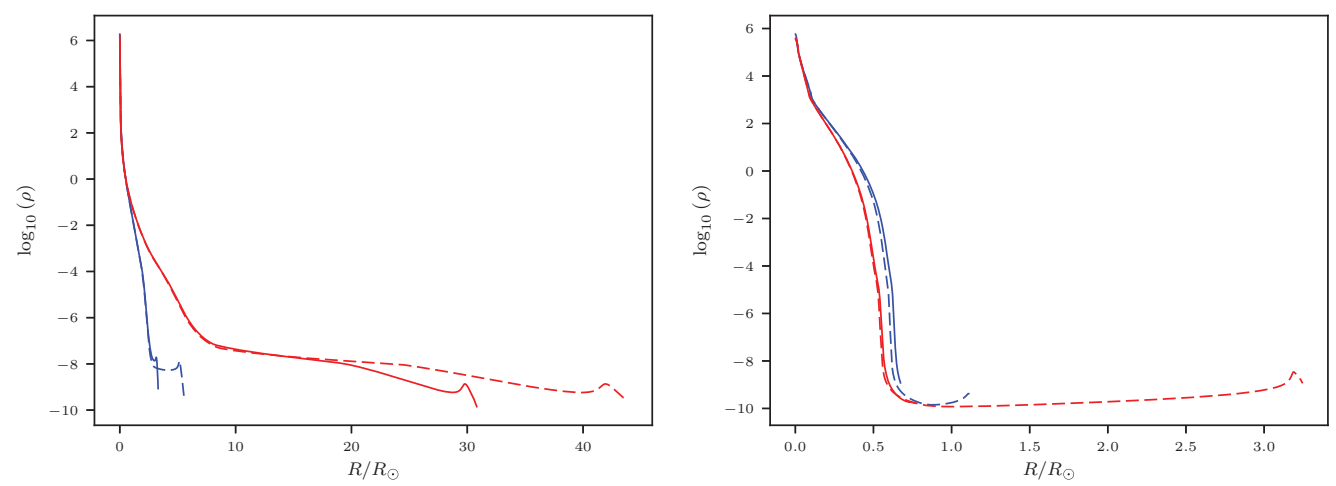

Figure 1. Left: Density profile of a $3 \mathrm{M}_{\odot}$ helium star model at the termination of evolution. The blue and red lines denote $Z=0.008$ and $Z=0.02$, respectively. Additionally, the solid and broken lines denote a clumping factor of $D=1$ and $D=10$, respectively. Right: The same for a model of mass $15 \mathrm{M}_{\odot}$. In all cases, $\beta=1$. 

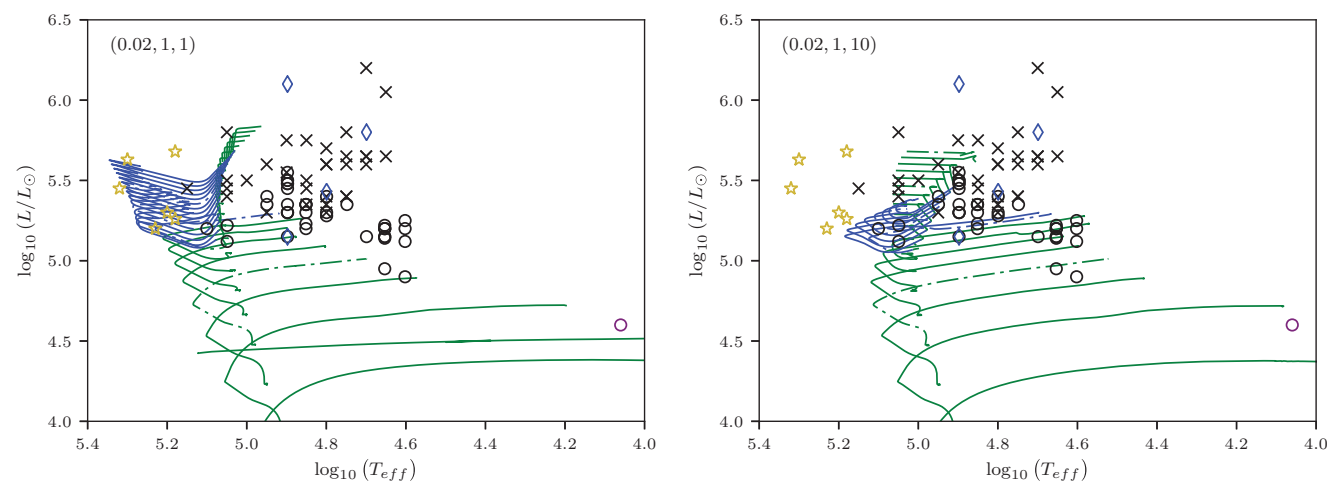

Figure 2. HR diagram of our evolved Galactic-metallicity models. For clarity, models with initial masses of $5,10,15$ and $20 \mathrm{M}_{\odot}$ are represented by dot-dashed lines. Observed WR star locations (see text for details) are marked as follows: WN, saltires; WC, circles; WO, yellow stars; and WN/WC transition objects, blue diamonds. Progenitor iPTF13bvn is marked with a purple circle (see Eldridge \& Maund 2016). All observed stars are hydrogen-free. The phase of WR mass loss is indicated, WN (solid, green line) and WC (solid, blue line); non-WR mass loss is shown with solid, black lines. Metallicity, mass-loss rate, and clumping factor are noted on the plots.

density with a large density inversion at the surface (see right panel of Fig. 1). The density inversion sits atop the extended envelope structure of the high-mass helium star models, and due to the stellar interior reaching the phase space of the iron-opacity peak Gräfener, Owocki \& Vink (2012), is affected by metallicity.

\section{Results}

We present our results for Galactic-metallicity models in Figure 2. Observational data is taken from Hamann et al. (2006) and Sander et al. (2012), for Galactic WN and WC stars; Hainich et al. (2014), for Large Magellanic Cloud (LMC) WN stars; and Tramper et al. (2013) and Tramper et al. (2015), for WO stars. Also included are results from Crowther et al. (2002) for LMC WC stars. In this work, we attempt to reproduce the observed locations of WR stars.

Galactic WN stars. The observed early-type WN stars lie near the HeZAMS for massive helium stars and are, generally, in good agreement with helium star models of initial masses above $\approx 10 \mathrm{M}_{\odot}$. However, the agreement is not so favourable for the observed late-type WN stars. These WN stars have stellar temperatures that are far cooler than those at the HeZAMS, and their locations cannot be reproduced by using models with $\beta>0$.

Without mass loss $(\beta=0)$, we see an interesting result: the higher mass helium star models do, indeed, cross the region of observed (hydrogen-free) late-type WN stars for solar metallicity ("old" and "new"). A small amount of mass loss will remove the outer layers of the envelope and expose the hot interior of the model; thus, without mass loss, the model swells due to inflation and the surface temperature decreases. The inclusion of envelope clumping results in cooler stellar temperatures, as expected. However, even with the inclusion of clumping, our helium star models are unable to reproduce the observed locations of the coolest Galactic WN stars if we use the standard WR mass-loss rates (i.e. $\beta=1$ ).

To summarise, the large radii of the WN stars is due to either a small amount of mass loss or clumping in the envelope. 


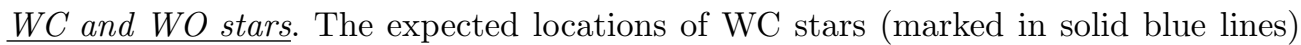
are in very poor agreement with the positions of observed WC stars. The standard evolutionary picture of WR evolution-suggested by Sander et al. (2012) where the WC phase succeeds a WN phase-is clearly insufficient to explain this discrepancy. We note from Fig. 2 that low-mass helium models can reproduce the observed locations of earlyand late-type WC stars.

We note the observed WO stars on the HR diagram are hotter and more luminous than the observed WC stars. A higher luminosity implies a higher stellar mass, and we indeed find the observed WO stars in a region predicted by our high-mass models. Though difficult to draw definitive conclusions due to the lack of observational evidence, we argue the standard description of WC stars used in evolutionary models is incorrect and actually applies instead to WO stars.

As can be seen in Fig. 2, the locations of the observed WC stars coincides with the locations of the low-mass helium giants. The surface composition identifies these lowmass helium giants as WN stars, not WC stars. Due to the weak mass-loss rates of the low-mass helium stars, nitrogen remains abundant on the stellar surface rather than being removed, as is the case for the higher mass stars. It is possible that the surface nitrogen may be removed by an alternative mechanism of extra mixing (Frey, Fryer \& Young 2013): nitrogen is mixed into the stellar interior and removed via nuclear processing.

We note that, by including clumping in our stellar models, our models evolve closer to locations on the HR diagram where WC stars are observed. However, the coolest WC stars still arise from the same low-mass helium giants. Furthermore, WO stars-which arise from the most massive stars - have much shorter lifetimes than the stars that we suggest are WC stars. This may explain why WO stars make up only a small fraction of the WR population.

In summary, our results suggest that both WC and WO stars are represented by models with $\beta=1$. However, they differ in terms of mass: WO stars are from the most massive of stars (an initial helium star mass of $\gtrsim 13 \mathrm{M}_{\odot}$ ), while WC stars come from less massive stars (an initial helium star mass of $\lesssim 13 \mathrm{M}_{\odot}$ ). Similar findings were made by Groh et al. (2013) and Sander et al. (2012). We note that adding clumping improves the agreement but we find that WO stars must be unclumped.

Binary Configurations. Radial expansion by way of a clumped envelope may affect the nature of binary interactions. The evolution of binary systems of helium stars will be analysed in a future paper. Figure 3 shows the initial conditions required for the system to experience a binary interaction - a Roche-lobe overflow. We see that, in general, helium binary systems do not interact, even with the application of enhanced inflation. For all metallicities, a WR binary system requires a period of less than a day to interact. In contrast, helium giants do interact quite readily due to their expansive envelopes.

\section{Discussion}

In light of our work (see McClelland \& Eldridge 2016), we can draw some firm conclusions about certain aspects of WR star evolution and speculate about others. We shall now discuss our conclusive findings.

First, WO stars are what we have always considered to be WC stars in stellar models. They are the progeny of the most massive WN stars $\left(M_{\mathrm{He}, \mathrm{i}}>8 \mathrm{M}_{\odot}\right)$ that have suffered significant mass loss and are the hottest WR stars.

Second, WC stars evolve from less massive stars $\left(M_{\mathrm{He}, \mathrm{i}}<8 \mathrm{M}_{\odot}\right)$ and are unlikely to be the evolutionary end-points of the typical observed WN star. The evolution of these 

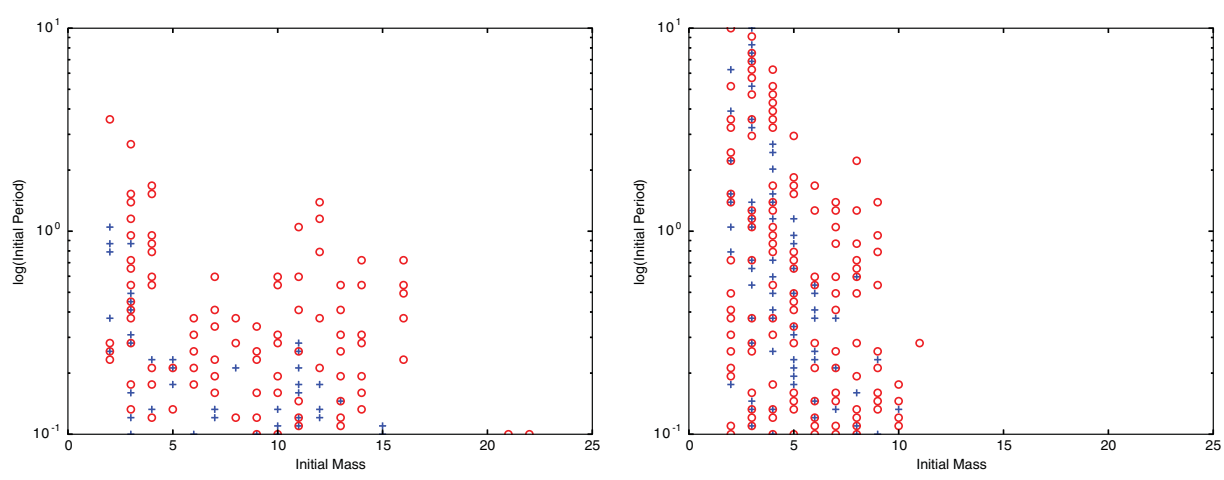

Figure 3. Initial period and initial mass of models that undergo a binary interaction. Left to right: $Z=0.008$ and 0.02 respectively. Red circles indicate a clumped envelope; blue crosses indicate an unclumped envelope.

stars could be described either as an inflationary effect occurring towards the end of their lives or as them becoming helium giants.

If the stellar envelope is clumped, it can only occur for WN stars. The only way to reproduce the locations of the late-WN stars on the HR diagram with standard-evolution models is to remove all mass loss, as even slight mass loss will drive a model to higher stellar temperatures. WC and WO stars do not require enhanced inflation to reproduce their HR diagram positions. The evolutionary models predict the locations of WO stars without a problem: a hot phase consequential to high-mass WN stars. The predictions for WC stars are correctly realised for the cooler temperatures of low-mass WC stars.

\section{References}

Asplund M., Grevesse N., Sauval A. J., Scott P. 2009, ARAA , 47, 481

Crowther P. A., et al., 2002, A\&A A, 392, 653

Crowther P. A., 2007, ARAA, 45, 177

Eggleton P. P. 1971, MNRAS, 151, 351

Eldridge J. J., Vink J. S. 2006, A\&BA , 452, 295

Eldridge, J. J. \& Maund, J. R. 2016, MNRAS, 461, L117

Frey L. H., Fryer C. L., Young P. A., 2013, ApJ(Letters), 773, L7

Gräfener G., Owocki S. P., Vink J. S., 2012, A $\mathcal{E} A$, 538, A40

Grevesse N., Sauval A. J. 1998, Space Sci. Revs, 85, 161

Groh J. H., Meynet G., Georgy C., Ekström S., 2013, A\& A, 558, A131

Hainich R. et al., 2014, A\&A A, 565, A27

Hamann W.-R., Gräfener G., Liermann A. 2006, A\&A, 457, 1015

McClelland, L. A. S. \& Eldridge, J. J. 2016, MNRAS, 459, 1505

Nugis T., \& Lamers H. J. G. L. M. 2000, $A \& \mathcal{G} A$, 360, 227

Sander A., Hamann W.-R., Todt H., 2012, A\& $A$, 540, A144

Stancliffe R. J., Eldridge J. J. 2009, MNRAS, 396, 1699

Tramper F. et al., 2013, A\& $A$, 559, A72

Tramper F., et al., 2015, in IAU Symposium, Vol. 307, IAU Symposium, pp. 144-145 